\title{
Seeded Growth of Mesoscale Quantum-Confined Semiconductor Nanoplatelets
}

Stephanie M. Tenney ${ }^{1}$, Lauren A. Tan ${ }^{1}$, Mikayla L. Sonnleitner ${ }^{1}$, Anthony V. Sica ${ }^{1}$, Ashley J. Shin ${ }^{1}$, Timothy L. Atallah², Justin R. Caram ${ }^{1 *}$

${ }^{1}$ Department of Chemistry and Biochemistry, University of California, Los Angeles, 607 Charles E. Young Drive, Los Angeles, California 90095-1569, United States

${ }^{2}$ Department of Chemistry and Biochemistry, Denison University, 500 West Loop, Granville, Ohio 43023, United States

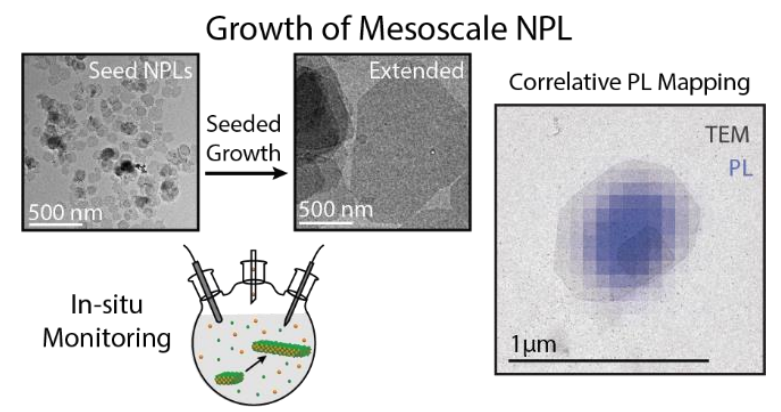

Colloidally grown (II-VI) semiconductor nanoplatelets display significantly smaller lateral dimensions than their mechanically exfoliated 2D van der Waal (vdW) semiconductor counterparts. Here, we show that a seeded growth procedure allows us to significantly extend the lateral area of atomically precise nanoplatelets to the mesoscale $\left(>1 \mu \mathrm{m}^{2}\right)$. Using CdTe nanoplatelets as a model system, we optimize reaction parameters to expand a variety of nanoplatelets with different thickness and compositions. In situ spectroscopy results demonstrate that large NPLs grow through a mechanism of lateral ripening of seeds. Correlative optical spectroscopy and electron microscopy measurements show that the photoluminescence displays resolvable spatial inhomogeneities, similar to 2D semiconductors. Overall, these mesoscale nanoplatelets can be analogized to vdW semiconductors, with the added advantages of scalable colloidal synthesis, thickness tunability and solution processability.

Keywords: nanoplatelet, seeded growth, cadmium telluride, nanocrystal, 2D

II-VI semiconductor nanoplatelets (NPLs) are a class of nanocrystals with atomically precise confinement in the thickness dimension. They show exceptional photophysical properties, including 
narrow linewidths, tunable emission, large absorption cross sections, and high quantum yields. ${ }^{1-3}$ They are also structurally versatile, able to vary in composition from $\mathrm{CdX}(\mathrm{X}=\mathrm{S}, \mathrm{Se}, \mathrm{Te})$ to $\mathrm{HgX}$, and are amenable towards lateral and longitudinal heterostructures-all while maintaining their discrete monolayer (ML) thicknesses..$^{1,4-8}$

Due to their similar anisotropic structure, a comparison can be made between NPLs and van der Waals (vdW) 2D materials. Both systems display strong exciton binding energy, dielectric screening by external environments, and thickness-dependent band structure. ${ }^{9-11}$ Additionally, NPLs may also show exotic properties characteristic of vdW materials, such as single photon emission, topological insulation, spin-orbit effects, and fractional quantum hall effects. ${ }^{12-14}$ Unlike conventional exfoliated vdW 2D materials that often display a finite thickness distribution, II-VI semiconductor NPLs may be prepared with monodispersed thickness through colloidal preparation, with direct bandgaps for all thicknesses, and chemical tunability through ligand design. ${ }^{15}$ Realizing the connections to $2 \mathrm{D} \mathrm{vdW}$ materials requires NPLs with sufficiently large lateral areas, especially to integrate them into single nanoplatelet devices or high-performance thin film devices. ${ }^{16}$

Prior work has shown CdX and HgX NPLs can be synthesized with lateral dimensions (longest edge) up to $\sim 500 \mathrm{~nm}$ using slow injection of precursors, although most procedures utilize fast injection that results in smaller lateral sizes of $100-200 \mathrm{~nm}$ or less. ${ }^{17}$ Studies of smaller NPLs reveal lateral size dependence that shows intriguing photophysical properties including an enhanced absorption cross section, giant oscillator strength, altered spontaneous and stimulated emission thresholds, modified biexciton quantum yield, and depressed Auger recombination. ${ }^{18-22}$ However, the absence of a standardized procedure to synthesize larger NPLs limits the exploration of such properties into bulk II-VI nanosheets, which may behave more similarly to 2D semiconducting materials than previously explored NPLs (area $<3000 \mathrm{~nm}^{2}$ ). Ultrathin nanosheets of other semiconducting materials have been synthesized, such as $\mathrm{Cu}_{2-\mathrm{x}} \mathrm{S},{ }^{23} \mathrm{In}_{2} \mathrm{Se}_{3}{ }^{24}, \mathrm{PbS}^{25}$, perovskite $\mathrm{CsPbBr}_{3}{ }^{26}$, and a host of transition metal dichalcogenides (TMDs). Despite this variety, robust control of large area II-VI nanosheets with optical properties akin to their highly-confined small area analogs has not been shown. 
To synthesize mesoscale NPLs, we employ a modified seeded growth method that has been used previously for both the growth of core-crown NPLs and the lateral extension of small CdSe NPLs. In a typical core-crown growth procedure, an amount of pre-synthesized NPLs are used as seeds and a second reaction introduces a chalcogen precursor of a different composition to yield a heterostructure. ${ }^{7,27-32}$ The extension method of small CdSe NPLs from $~ 300$ to $4000 \mathrm{~nm}^{2}$ is similar, but it uses the same precursor compositions injected into a solution of NPL seeds at slower rates. ${ }^{33-35}$ We build on these procedures in our seeded growth, but carefully explore the parameters to yield NPLs that are more than double in size while maintaining their size-confinement in the thickness dimension.

We show that careful control of the reaction parameters which affect 2D NPL growth allows us to demonstrate the optimal conditions for mesoscale CdTe NPLs and investigate how the NPL population evolves during the seeded growth process. We then utilize correlative imaging of single NPLs using a combination of fluorescence and electron microscopy to show that large NPLs exhibit lateral heterogeneity in their photoluminescence. Overall, this study demonstrates that mesoscale II-VI NPLs can possess differing electronic structure within a single NPL and represent a new class of colloidal 2D semiconductors rather than confined single emitters. Finally, the advent of mesoscale 2D vdW materials has enabled a generation of novel electronic devices as a result of their microscopic characterization and control, and a similar application space may exist for colloidal NPLs. ${ }^{36-38}$ Their size may make them similarly amenable to direct microscopic manipulation and could pave the way for more advanced, single nanocrystal devices derived from colloidal confined II-VI semiconductors.

\section{Results and Discussion}


a)
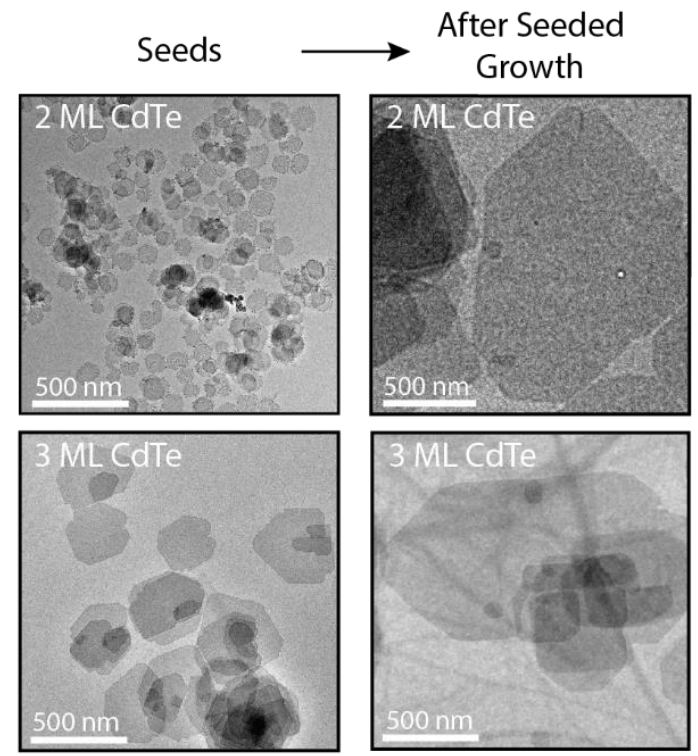

b)

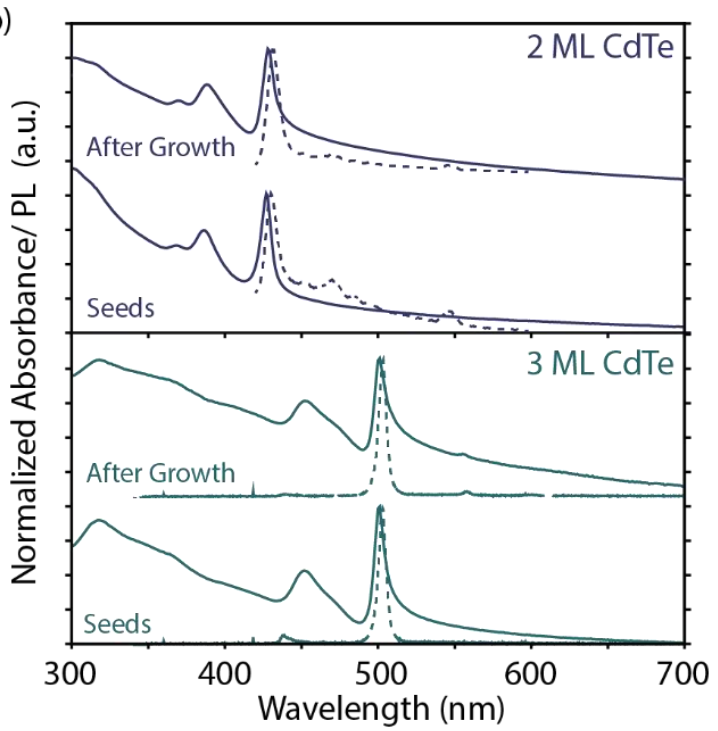

Figure 1. a) TEM of 2 and 3 ML CdTe NPL seeds before and after seeded growth. b) Absorption (solid lines) and photoluminescence (PL, dashed lines) spectra before and after seeded growth.

We start by showing two exemplars of the seeded growth process in Figure 1. Following literature procedures, we produce 2 and 3 monolayer (ML) CdTe seeds with an average lateral extent of $93.3 \pm 2.1 \mathrm{~nm}$ and $367 \pm 11 \mathrm{~nm}$ respectively (results of size counting in Table S1). ${ }^{4-6} 2 \mathrm{ML}$ CdTe seed NPLs are synthesized by a fast injection of trioctylphosphine telluride (TOP-Te) into a cadmium propionate solution at $180{ }^{\circ} \mathrm{C}$. For $3 \mathrm{ML}$ seed NPLs, we follow a slow injection method, where TOP-Te is continuously injected into a solution of cadmium propionate at $210{ }^{\circ} \mathrm{C}$. All NPL products are 
then centrifuged and resuspended in fresh solvent to be used as seeds for subsequent growth. We then extend these NPL seeds to lateral sizes of $1000-1500 \mathrm{~nm}$ by reintroducing them to a second, slower injection of new chalcogen precursor and carefully control the rate of growth (further details in Methods). Despite the change in size, the larger NPLs retain their absorption and emission profiles after this seeded growth process (Figure 1b), suggesting no change in thickness with increase in lateral size.

a)

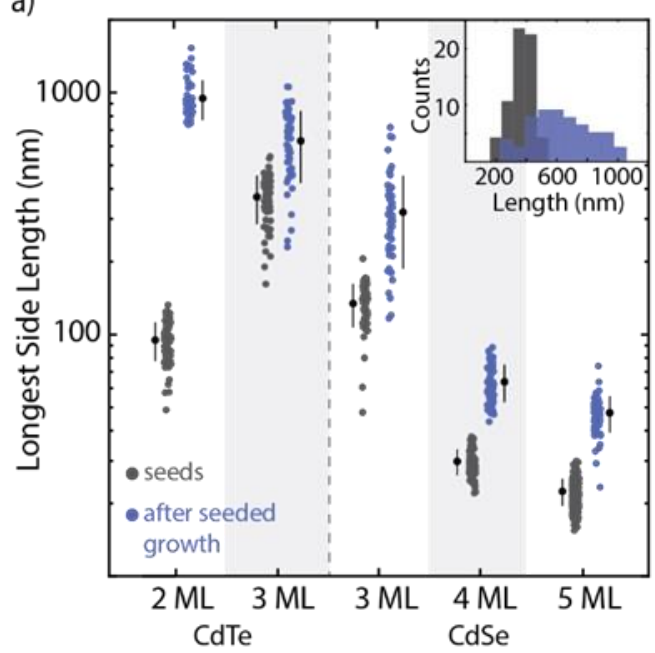

b)
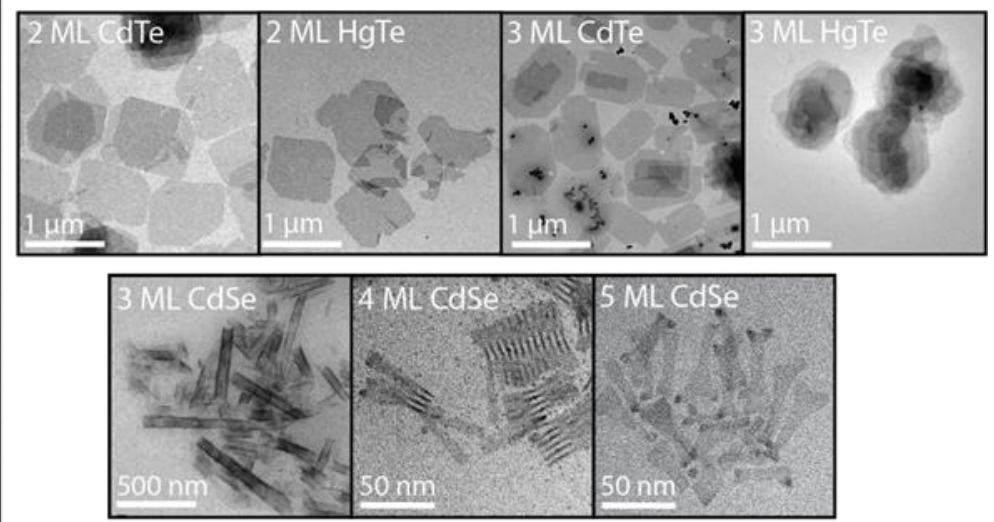

Figure 2. a) The difference in lateral sizes before (grey) and after (blue) seeded growth for various CdX NPL thicknesses. Solid colored circles represent the counts for each size distribution, while the solid black circles show the mean and standard deviation of each condition explored. Inset shows an example of the full histogram of sizes before (grey) and after (blue) seeded growth for 3 ML CdTe. b) Representative TEM images of NPLs after seeded growth, including HgTe from cation exchange with large CdTe.

In Figure 2, we demonstrate the scope of this procedure by applying the conditions of seeded growth to various compositions, specifically CdTe NPLs (2-3 ML) and CdSe NPLs (3-5 ML), and measure the change in lateral extent using TEM (Figure 2a). We observe the greatest enhancement in CdTe NPLs, but all materials show a significant change of at least twice the mean size while maintaining 
their absorption and emission (Figure S2 and Table S1). We note that, in contrast to the large CdTe, CdSe remain smaller, more rectangular, and show emission from defects or other NCs after seeded growth (Figure S2). We hypothesize that slightly differing synthetic procedures, higher nucleation barriers, and/or surface strain properties result in smaller CdSe NPLs. ${ }^{39-41}$ In Figure 2b, we show representative TEM of the CdTe and CdSe NPLs after seeded growth as well as extended HgTe NPLs (additional TEM images used for counting are shown in Figure S3). Extended 2 and 3 ML CdTe can be isolated after seeded growth and introduced to a solution of mercury acetate dissolved in oleylamine at room temperature to yield these large HgTe NPLs through a cation exchange process (further details in Methods). This demonstrates that large CdTe NPLs are robust enough to undergo a complete cation exchange while maintaining their large-area morphology and thickness, giving access to mesoscale NIRemissive NPLs (Figure S4). 
a)

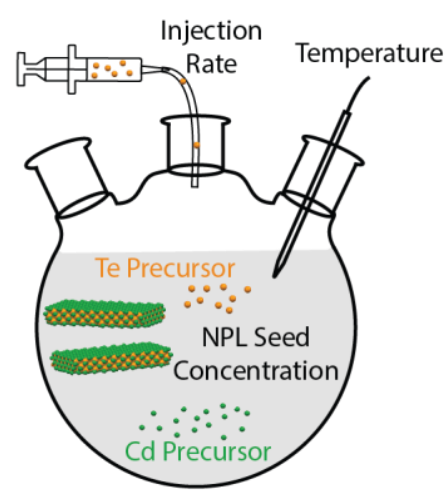

b)
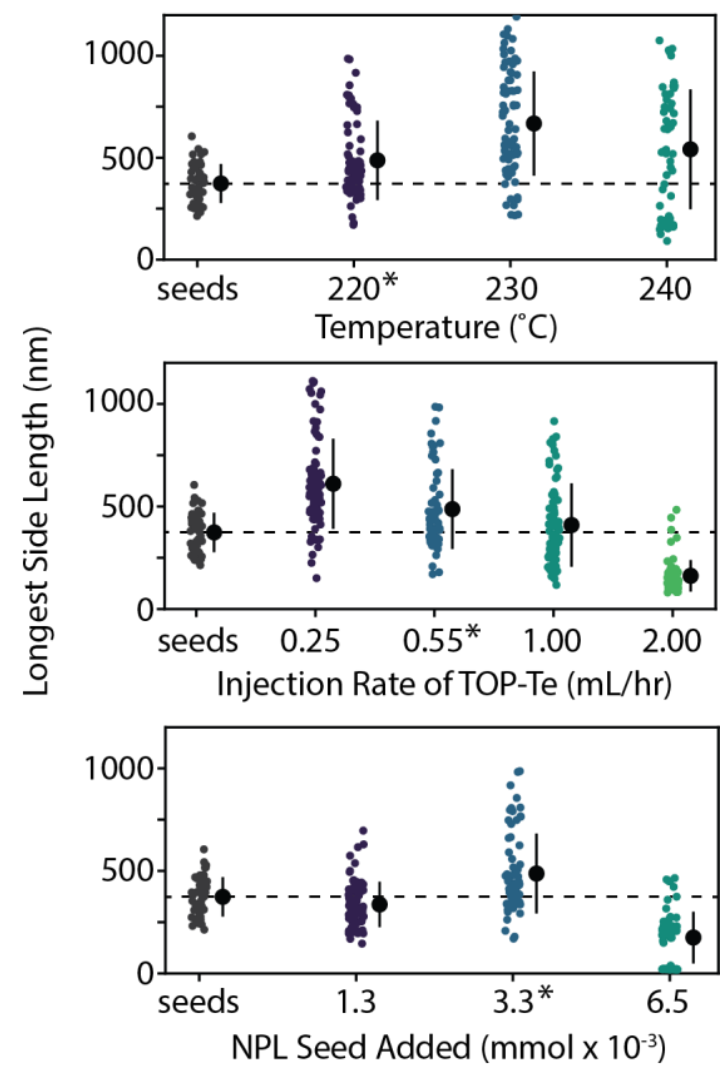

Figure 3. a) Experimental diagram of explored reaction conditions and b) size distributions of the longest edge of NPLs after seeded growth (measured from TEM images, Figure S5) compared to the seeds. Solid colored circles represent the counts for each size distribution, while the solid black circles show the mean and standard deviation of each condition explored. The dashed line represents the average size of the initial seeds. The $(*)$ indicate control conditions when changing other variables independently. 
To demonstrate that the seeded growth process is necessary, we performed two control experiments — slow injection without seeds and fast injection with seeds — and both produced NPLs less than $\sim 500 \mathrm{~nm}$ in size along with many 3D nanocrystals (Figure S6). We then hypothesized that the same parameters which govern 2D NPL nucleation and growth including temperature, injection rate, and precursor concentration are important for controlling size during seeded growth and can be tuned to optimize the procedure. ${ }^{33,39,42}$ Additionally, these parameters are important for maintaining a regime of growth which outcompetes the critical supersaturation for nucleation and suppresses secondary nucleation..$^{33,43,44}$ We indeed find that the largest NPLs occur within a narrow range of optimized conditions and show how the experimental parameters of the slow injection seeded growth affect the extension in 3ML CdTe by isolating each variable individually (Figure 3). First, slightly elevated temperatures relative to the initial seed growth $\left(210{ }^{\circ} \mathrm{C}\right)$ result in large extended NPLs, however this also results in a broadening of the size distribution. Beyond $230{ }^{\circ} \mathrm{C}$, NPLs smaller than the seed NPLs nucleate during the growth (secondary nucleation). Second, the injection rate must be slowed significantly in order to favor large NPL growth $(0.55 \mathrm{~mL}$ or $0.055 \mathrm{mmol} / \mathrm{hr}$ compared to $5 \mathrm{~mL}$ or $0.25 \mathrm{mmol} / \mathrm{hr}$ in a typical 3 ML CdTe NPL synthesis), but must still be high enough to maintain the kinetic instability driving 2D growth. ${ }^{39}$ Too slow of an injection rate $(<0.55 \mathrm{~mL} / \mathrm{hr}$ of $0.1 \mathrm{M}$ TOP-Te $)$ forms many $3 \mathrm{D}$ agglomerates alongside the NPLs (Figure S5), while too fast (> $1 \mathrm{~mL} / \mathrm{hr}$ ) forms primarily secondary NPLs. Finally, the concentration of seeds affects the amount of edge sites for monomers to be added- too low results in few extended NPLs while too high results in clumps of large NPLs, both of which lead to many secondary NPLs. The conditions that we use for further spectroscopic investigation are indicated by the (*) in Figure $3 b$. 
a)

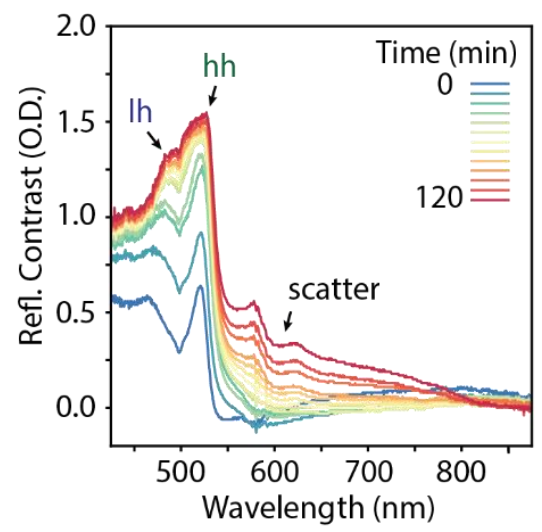

d)

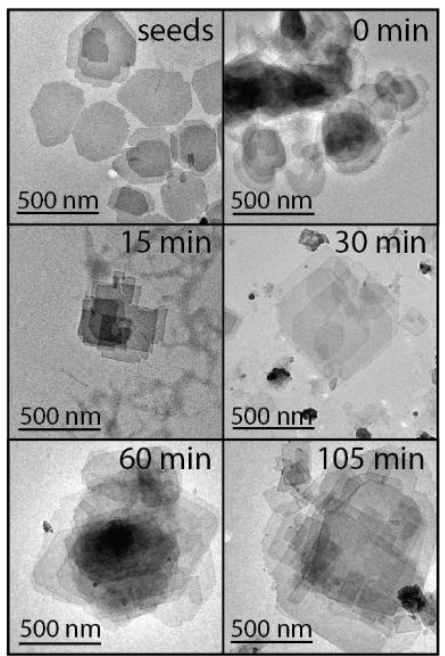

b)

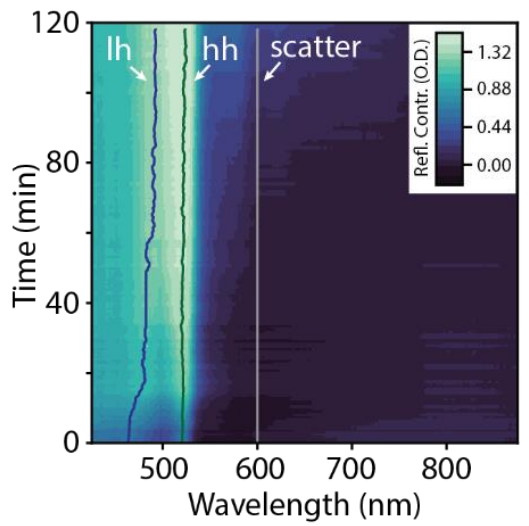

c)

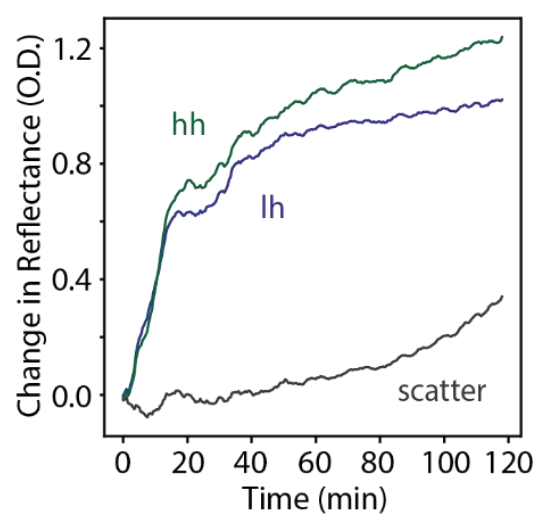

e)

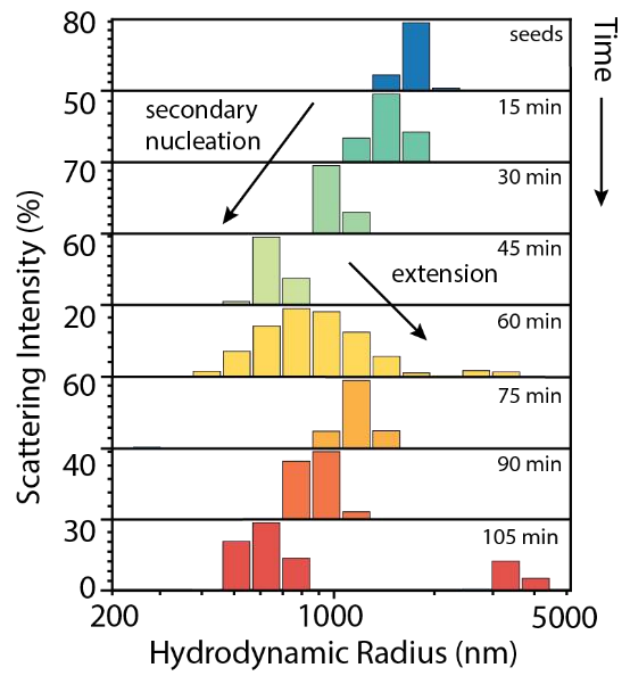

Figure 4. a) Reflectance contrast collected in situ (data shown for every 10 minutes) for the duration of a seeded growth of $3 \mathrm{ML}$ CdTe. b) The reflectance contrast change over time, sampled every $30 \mathrm{~s}$ with slices taken at the heavy hole (hh), light hole (lh), and $600 \mathrm{~nm}$ (scatter). c) The time slices are shown in more detail, highlighting the evolution of the excitonic transitions during seeded growth. d) TEM during early stages of growth starting with seeds before any precursor was added. e) Dynamic light scattering (DLS) of aliquots taken every 15 minutes during seeded growth of 3 ML CdTe.

To understand how NPL growth is occurring during this process, we now explore the reaction dynamics as a function of time in Figure 4. We perform in situ and ex situ spectroscopy and microscopy on our optimized seeded growth reaction $\left(0.55 \mathrm{~mL} / \mathrm{hr}\right.$ injection rate of $0.1 \mathrm{M}$ TOP-Te at $\left.220{ }^{\circ} \mathrm{C}\right)$. Using a 
reflectance probe to monitor changes in optical density (OD), we track changes in the light hole (lh) and heavy hole (hh) excitonic features as well as the scatter profile (at $600 \mathrm{~nm}$ ) over the course of a $2 \mathrm{hr}$ growth period (Figure 4a-b, more details in Figure S7). Their evolution is highlighted by taking slices of the spectra over time (Figure 4c). We interpret the increase in OD of the excitonic features to indicate the formation of new platelet area (either small secondary platelets or extension of the seeds), while we hypothesize that the scatter mostly informs about the formation of the larger platelet structures. We motivate our assignments by noting that scatter increases non-linearly with size of NPL, while absorption is proportional to the number of unit cells in the semiconductor - allowing us to distinguish between the two despite a lower concentration of large NPLs. ${ }^{45}$ These assignments allow us to qualitatively follow the reaction as a function of time. We observe two major growth periods of the excitonic features - a rapid initial increase from 0-20 min and another smaller increase from 30-45 min. Beyond this point, the change in OD is slow and continual. The scatter profile at $600 \mathrm{~nm}$ shows an oscillatory behavior that appears anticorrelated to the excitonic features during the first hour. We also note the growth of thicker NPL species (indicated by absorption at 550 and $650 \mathrm{~nm}$ ) after $60 \mathrm{~min}$, but they are not as significant after the reaction is quenched and the nanoplatelets are centrifuged and resuspended. Thicker NPLs appear in relatively low concentration compared to the 3 ML NPLs (Figure S8).

In order to understand the morphological changes in the NPLs during seeded growth, we utilize TEM and dynamic light scattering (DLS) to track the populations of seeds and secondary NPLs during the reaction. In Figure 4d, we show TEM of the largest platelets during the stages of growth. Before any TOP-Te has been injected ( $0 \mathrm{~min})$, there is a slight dissolution around the edges of the NPLs, which can explain the very slight loss of OD during the first few minutes. Once the injection has begun, however, we see the edges fill out and become rectangular. At $30 \mathrm{~min}$, we observe secondary nucleation starting to occur alongside a noticeable extension in the larger NPLs. Interestingly, this extension does not appear to be continuous during the last 30 minutes - a nucleation burst of many small NPLs is noticeable by 45 min, and only after that burst are the largest NPLs formed (60 min). 
The composition of seeds and secondary NPLs are homogenous, and we cannot use the excitonic features to distinguish between the two populations. To get an ensemble measure of NPL behavior, we use DLS (Figure 4e, more details in Supporting Information) to obtain insight into the change in total population, both small and large. This results in a relative size measurement that avoids counting bias and indicates the presence of multiple size distributions (Figure 4e). We note that the hydrodynamic radius is derived for a spherical particle and does not reflect the absolute size of the NPLs, but DLS has been used with good agreement to TEM sizes for small NPLs ( 10-100 nm lateral sizes). ${ }^{46-48}$ Our CdTe NPLs are laterally much larger so we do not expect quantitative agreement with TEM size as we do not correct for their greater anisotropy in size. We observe that from 0-45 min, the overall population distribution is decreasing in size, which is explained by the nucleation of secondary small NPLs. At 60 min, there is some scattering from larger particles, which coincides with the appearance of the $\sim 1 \mu \mathrm{m}$ NPLs. Continuing the growth beyond an hour continues with yet another nucleation burst of smaller NPLs, while it seems that the large NPLs have reached a maximum size between 1000-1500 nm and do not continue to extend. The small secondary NPLs appear to become stable and large enough to extend themselves at this point, and reach $\sim 500 \mathrm{~nm}$ by $2 \mathrm{hr}$ (Figure S9)—limiting the maximum size of the larger seeded NPLs.

By connecting the results from reflectance, TEM, and DLS to describe what is occurring during the seeded growth process, we see stepwise or oscillatory changes in the spectra and size that correlate to secondary nucleation bursts followed by a slowed growth period which results in extension of the seed NPLs. This suggests that the lateral extension is not a continual addition of CdTe unit cells but rather can be explained through the balance of nucleation, growth, and ripening that has been described in the literature. ${ }^{42,49-51}$ First, the seed NPLs are introduced to the flask at elevated temperatures and begin to dissolve around the edges. Without any CdTe monomer present, the NPLs are unstable at high temperatures and detachment of CdTe occurs around the edges. As the slow injection of precursors begins, growth occurs steadily as the monomer concentration builds up. Eventually a critical concentration threshold is reached, and a burst of rapid secondary nucleation occurs-depleting the 
available monomer in solution. This then causes the smaller area NPLs to become unstable and dissolve, donating their monomers to the larger, more thermodynamically stable seed NPLs. ${ }^{39,49}$ In a traditional fast injection NPL synthesis, the monomer would then continue to be used up until the thinner NPLs are no longer stable, and dissolve onto the next thicker nuclei. ${ }^{49}$ In our experiments, the constant injection of monomer maintains the high concentration and prevents major shifts towards thicker NPLs. Instead, we observe repeating cycles of nucleation and extension within the same thickness to obtain significantly larger platelets. These observed growth stages can be replicated in seeded growth trials with differing injection rates. When comparing concentration of precursor added, rather than time, we see the stages occurring similarly at certain concentration thresholds (Figures S10and S11).

a)

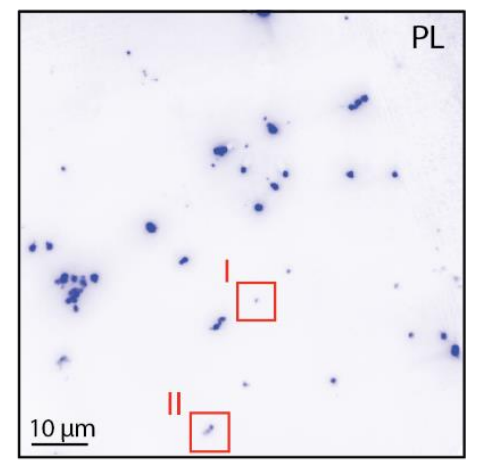

b)

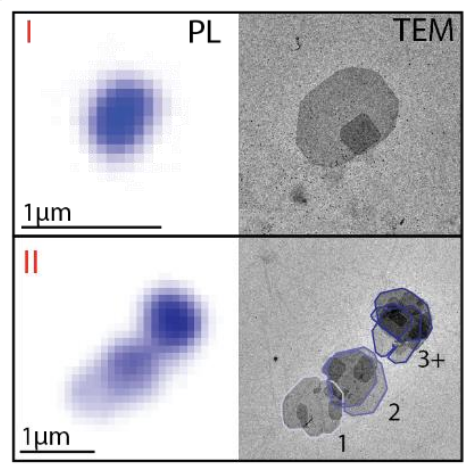

c)

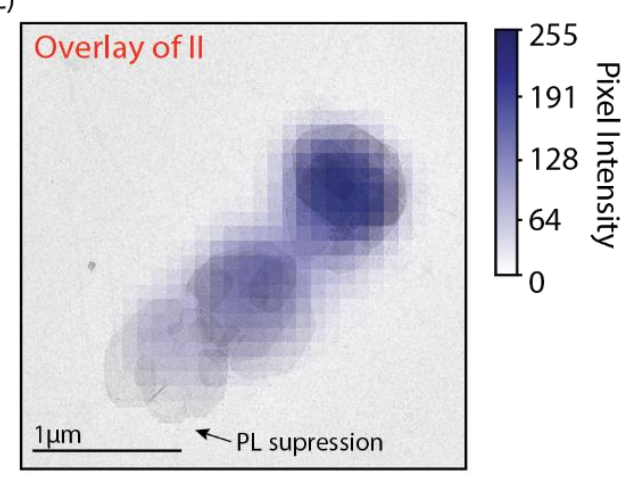

Figure 5. a) Photoluminescence imaging of large 3 ML CdTe NPLs, showing a single NPL (I) and a group of NPLs (II). b) The TEM for spots (I) and (II) correlated to their PL, with the domains of 1, 2, and 3+ NPLs marked in (II). c) Overlayed PL and TEM for spot (II), showing spatial heterogeneity of PL in the single NPL, as well as the integer increase in pixel intensity as size of stack increases.

The seeded growth method allows us to reproducibly synthesize mesoscale NPLs, and we turn our efforts next to spectroscopy to further study their photophysical properties. To confirm the presence of dispersed platelets, we perform correlative light and electron microscopy. Correlative imaging has been used previously on nanocrystals and 2D materials to study photophysical properties such as plasmonic behavior, catalysis, and defect emission in relation to their structure. ${ }^{52-56} \mathrm{We}$ adapt these methods here to perform photoluminescence (PL) mappings of our large NPLs. In Figure 5, we demonstrate that 
mesoscale single NPL structures can be observed under conventional light and fluorescence microscopy (similar to their TMD counterparts). The lateral extent of our mesoscale NPLs allows us to resolve PL beyond the point spread function and the entire NPL can be visualized with standard wide-field microscopy. This is advantageous for studying NPLs because confirming the isolation of single NPLs in the literature relies on resolving fluorescence blinking or single photon emission (through second order photon correlations) - properties of which may not be universal across different compositions and sizes. ${ }^{3,21}$ By imaging the PL over the entire NPL structure, we utilize a method that allows for further studies of singe crystal NPL dynamics.

To correlate light and electron microscopy, large NPLs obtained through seeded growth are diluted and drop cast onto a TEM grid with an alphanumerical reference pattern. The grid is then sealed between microscope slides and the PL is imaged using an inverted microscope equipped with an sCMOS camera (more details in Figure S1). Figure 5a shows the PL for a square within the grid, along with closeups (Figure 5b spots (I) and (II)) correlated to TEM images. The image alignment process is described in Figure S12, along with an additional example of correlation with the same methods applied. We can resolve the entire structure of a single NPL that is bright and does not contain any large tears (Figure 5b, (I)), and we also examine a set of NPLs (Figure 5b (II)) to study defects and NPL stacking.

For image (II), when the PL and TEM images are overlayed (Figure 5c), we observe spatial heterogeneity within the lower, single NPL. The lower half of the NPL is non-emissive and may be defective from the large tear. A closer examination (Figure S13) shows that PL is suppressed around the edges and screw dislocation, where there may be more defects. These results draw comparison to $2 \mathrm{D}$ semiconductors - lateral heterogeneity of PL in 2D semiconductors can be demonstrated from many effects including defects, strain, chemical heterogeneity, and manipulation by 0D-2D heterostructures. ${ }^{57-64}$ These vdW materials exhibit localized electronic structure changes that do not quench the entire nanostructure and can be spatially resolved through PL mapping. The heterogeneity of PL in our large NPLs contrasts them with smaller NPLs which have not demonstrated this behavior. Direct application into single crystal devices will allow for further studies of mesoscale NPL properties. 
As additional demonstrations of our NPL imaging technique, the number of NPLs within a stack can be resolved using the intensity of the imaged PL (Figure 5b-c), as shown by the near integer increase in the intensity that correlates to 1,2, and 3+ NPLs — confirmed by TEM. We also collect PL lifetimes and observe variability depending on the number of NPLs within isolated stacks (Figure S14). With increasing number of NPLs the average lifetime decreases, which is consistent with a change in nonradiative pathways due to energy transfer amongst the stack and the increased likelihood of exciton trapping by a nonemissive NPL. ${ }^{65,66}$

\section{Conclusions}

We demonstrate the controlled growth of mesoscale II-VI semiconductor NPLs which reach areas up to $2.25 \times 10^{6} \mathrm{~nm}^{2}$ for the first time. We confirm our understanding of NPL growth by applying the parameters that drive 2D extension towards the development of a seeded growth process for CdTe NPLs. In situ methods allow us to study the competing growth between secondary nucleation and lateral extension during this process. We also show that this seeded growth procedure can be applied to NPLs of different thicknesses and compositions, with large extensions in 2-3 ML CdTe (as well as HgTe after cation exchange) and limited extension in 3-5 ML CdSe. These mesoscale NPLs have large areas while maintaining thickness confinement that allow them to uniquely contain properties of both quantum dots and $2 \mathrm{D}$ materials, showing promise in single nanocrystal devices. In contrast to more traditional $2 \mathrm{D}$ materials, these NPLs are amenable to the wide variety of colloidal synthetic techniques (cation exchange and crown/shell growth, ligand exchanges, etc.) to develop new and interesting interfaces and may benefit from improved electronic properties through surface ligand protection. Finally, their lateral sizes allow us to apply a method of PL imaging across the entire NPL and correlate it to structure via TEM. Through this method we isolate single NPLs and show that mesoscale NPLs have properties similar to 2D semiconductors-demonstrating a new class of material to bridge the gap between quantum-confined nanocrystals and 2D materials.

\section{Methods}




\section{Materials}

Cadmium acetate dihydrate (Acros, 98\%), selenium powder (Acros, 99.5\%), tellurium powder (Acros, 99.8\%), cadmium oxide (Alfa Aesar, 99.95\%), cadmium bromide tetrahydrate (Acros, 98\%) oleic acid (Alfa Aesar, 99\%), tri-n-octylphosphine (TOP) (Alfa Aesar, 90\%), 1-octadecene (ODE) (Alfa Aesar, 90\%), mercury (II) acetate (Chem-Impax International, 98.0\%), ethanol (Fisher, 95.27\%), hexanes (Fisher, 98.5\%), toluene (Alpha Aesar, 99.8\%), propionic acid (Fisher), and oleylamine (Tokyo Chemical, $50.0 \%)$ were used.

\section{Synthesis of Precursors}

Cadmium propionate $\left(\mathrm{Cd}(\text { prop })_{2}\right) .1 .036 \mathrm{~g}$ of $\mathrm{CdO}$ powder was mixed with $10 \mathrm{~mL}$ propionic acid under Ar flow for 1 hour. The flask was then heated at $140{ }^{\circ} \mathrm{C}$ after opening to atmosphere in order to reduce the volume by half. The white solution was precipitated with acetone and centrifuged. The supernatant was discarded, and the solid was dried and stored in a vacuum desiccator.

TOP-Te $(1 \mathrm{M})$. Following Izquierdo et al., ${ }^{4}$ In a small flask, $0.254 \mathrm{~g}$ of Te powder and $2 \mathrm{~mL}$ of tri-noctylphosphine were degassed under vacuum at room temperature. Then, under Ar flow, the solution was stirred at $275{ }^{\circ} \mathrm{C}$ until the dissolution was complete, and the solution turned yellow. The solution was cooled and stored under Ar.

2 Monolayer CdTe Seed Nanoplatelets. Following Pedetti et al., ${ }^{4,17} 170 \mathrm{mg}$ cadmium propionate, $104 \mu \mathrm{L}$ oleic acid, and $10 \mathrm{~mL}$ octadecene (ODE) were degassed for $1 \mathrm{hr}$ at $90{ }^{\circ} \mathrm{C}$. Under Ar flow, the temperature was increased to $180{ }^{\circ} \mathrm{C}$ and $130 \mu \mathrm{L}$ of $1 \mathrm{M}$ TOP-Te diluted in $650 \mu \mathrm{L}$ ODE was swiftly injected. After 20 minutes, $1 \mathrm{~mL}$ of oleic acid was added, and the reaction was cooled to room temperature. The crude mixture was precipitated with ethanol and centrifuged twice, then resuspended in $10 \mathrm{~mL}$ hexanes.

3 Monolayer CdTe Seed Nanoplatelets (slow injection). Adapted from Izquierdo et al., ${ }^{4,5} 260 \mathrm{mg}$ of cadmium propionate, $160 \mu \mathrm{L}$ of oleic acid, and $20 \mathrm{~mL}$ of $\mathrm{ODE}$ were degassed under vacuum at $90{ }^{\circ} \mathrm{C}$ for $1 \mathrm{hr}$. Under Ar flow, the reaction was heated to $210^{\circ} \mathrm{C}$ and $0.200 \mathrm{~mL}$ of $1 \mathrm{M}$ TOP-Te mixed with $3.75 \mathrm{~mL}$ 
of octadecene was injected with a syringe pump at a rate of $5 \mathrm{~mL} / \mathrm{hr}$. Once the injection was complete, $0.500 \mathrm{~mL}$ of oleic acid was injected and the solution was cooled to room temperature. The nanoplatelets were precipitated with $15 \mathrm{~mL}$ hexanes and $15 \mathrm{~mL}$ ethanol, centrifuged, and resuspended in $20 \mathrm{~mL}$ hexanes.

3 Monolayer CdSe Seed Nanoplatelets (slow injection). Adapted from Izquierdo et al., ${ }^{4,5} 240 \mathrm{mg}$ of cadmium acetate dihydrate, $0.150 \mathrm{~mL}$ of oleic acid and $15 \mathrm{~mL}$ of ODE were degassed under vacuum at 80 ${ }^{\circ} \mathrm{C}$ for $1 \mathrm{hr}$. Under Ar flow, the reaction was heated to $195{ }^{\circ} \mathrm{C}$ and $0.4 \mathrm{~mL}$ of $1 \mathrm{M}$ TOP-Se mixed with 3.75 $\mathrm{mL}$ ODE was injected with a syringe pump at a rate of $5 \mathrm{~mL} / \mathrm{hr}$. Once the injection was complete, the solution was cooled to room temperature and the nanoplatelets were precipitated with $15 \mathrm{~mL}$ hexanes and $15 \mathrm{~mL}$ ethanol, centrifuged, and resuspended in $20 \mathrm{~mL}$ of hexanes.

4 Monolayer CdSe Seed Nanoplatelets. Following Pedetti et al. ${ }^{27} 340 \mathrm{mg}$ cadmium myristate, $24 \mathrm{mg}$ Se powder, and $30 \mathrm{~mL}$ ODE were degassed for 20 minutes at room temperature. The temperature was then increased to $240{ }^{\circ} \mathrm{C}$ under Ar flow. When it reached $205{ }^{\circ} \mathrm{C}, 80 \mathrm{mg}$ of cadmium acetate were added, and the solution turned red. Then when the solution reached $240{ }^{\circ} \mathrm{C}$ it was left to react for 12 minutes followed by the addition of $2 \mathrm{~mL}$ oleic acid and rapid cooling to room temperature. The crude reaction mixture was added to $30 \mathrm{~mL}$ hexanes and $40 \mathrm{~mL}$ ethanol and centrifuged at $6000 \mathrm{rpm}$ for $10 \mathrm{~min}$, followed by a resuspension in $10 \mathrm{~mL}$ hexanes.

5 Monolayer CdSe Seed Nanoplatelets. Following Pedetti et al, ${ }^{27} 340 \mathrm{mg}$ cadmium myristate and $28 \mathrm{~mL}$ ODE were degassed for $20 \mathrm{~min}$ at room temperature. Under Ar flow, the solution was heated to $250{ }^{\circ} \mathrm{C}$ at which point $24 \mathrm{mg}$ of Se powder suspended in $2 \mathrm{~mL} \mathrm{ODE}$ was swiftly injected. After $30 \mathrm{~s}, 300 \mathrm{mg}$ of cadmium acetate was also added. The reaction was held at $250{ }^{\circ} \mathrm{C}$ for an additional $7 \mathrm{~min}$, followed by the addition of $1 \mathrm{~mL}$ oleic acid and rapid cooling to room temperature. The crude reaction mixture was added to $30 \mathrm{~mL}$ hexanes and $40 \mathrm{~mL}$ ethanol and centrifuged at $6000 \mathrm{rpm}$ for $10 \mathrm{~min}$, followed by a resuspension in $10 \mathrm{~mL}$ hexanes. Further purification was done by adding 1:4 ethanol to hexanes and centrifuging at $6000 \mathrm{rpm}$. 


\section{Seeded Growth}

In a three-neck round bottom flask, $65 \mathrm{mg}$ of cadmium propionate $(0.25 \mathrm{mmol}), 0.040 \mathrm{~mL}$ of oleic acid, and $5 \mathrm{~mL}$ of octadecene were degassed under vacuum at $80{ }^{\circ} \mathrm{C}$ for $20 \mathrm{~min}$. Under Ar flow, the solution was heated to a target growth temperature between $200-260{ }^{\circ} \mathrm{C}$ and $0.250 \mathrm{~mL}$ of $\sim 13 \mathrm{mM} \mathrm{CdX}$ seeds (volume adjusted to match concentrations across different thicknesses) were injected when the temperature reached $200{ }^{\circ} \mathrm{C}$. Once at the final temperature $\left(10{ }^{\circ} \mathrm{C}\right.$ warmer than the normal synthesis temperature), a solution of $0.050 \mathrm{~mL}$ of $1 \mathrm{M}$ TOP-Te mixed with $0.500 \mathrm{~mL}$ of ODE was injected with a syringe pump at $0.55 \mathrm{~mL} / \mathrm{hr}$. Immediately after the injection was complete, $0.250 \mathrm{~mL}$ of oleic acid was injected, and the solution was cooled. The nanoplatelets were then centrifuged at $9000 \mathrm{rpm}$ for $5 \mathrm{~min}$ and resuspended in $10 \mathrm{~mL}$ hexanes.

\section{Cation Exchange}

In a round-bottom flask, $0.240 \mathrm{~mL}$ of large CdTe NPLs and $6.0 \mathrm{~mL}$ of hexanes were mixed. Then, 0.600 $\mathrm{mL}$ of $10 \mathrm{mM} \mathrm{Hg}(\mathrm{OAc})_{2}$ in oleylamine was added and stirred at room temperature for $3.5 \mathrm{hr}$, monitored by the absorption of the light and heavy holes. Once complete, the nanoplatelets were then centrifuged at $9000 \mathrm{rpm}$ for 5 minutes and resuspended in $5 \mathrm{~mL}$ hexanes.

\section{Characterization}

Absorption spectra were acquired with an Agilent Cary 60 UV-Vis spectrophotometer. Photoluminescence (PL) was collected using an Ocean Optics Flame-S-VIS-NIR-ES Spectrometer and a Horiba Scientific PTI QuantaMaster400 Spectrometer. Reflectance measurements were collected using an Ocean Insight QR200-12-MIXED reflectance probe and an Avantes DH-2000 UV-VIS-NIR light source. Dynamic light scattering (DLS) was collected using a Coulter Beckman Dynamic Light Scattering Analyzer - N4 Plus at an angle of 62.6 degrees. 
Transmission electron microscopy (TEM) images were acquired with a FEI Tecnai T12 120kV TEM. The nanoplatelets were diluted in hexanes and drop casted on Ted Pella, Inc. carbon/formvar 300 mesh copper grids.

Photoluminescence imaging experiments were conducted on a home-built optical microscope. All NPLs were excited using a $405 \mathrm{~nm}$ pulsed diode laser (LDH-P-C-405 PicoQuant) with a power of 0.15 microwatts. The NPL sample slide is fixed to a piezo stage (Mad City Labs Nano-View/M 200-3), and the epifluorescence is sent through a 100x oil objective (Nikon MRD01905) and filtered using a $425 \mathrm{~nm}$ longpass dichroic filter. Wide-field images were collected using an sCMOS camera (Zyla sCMOS, Andor) (Figure S1b). Acquisition time was on average 10s.

For time correlated single photon counting, epifluorescence of the sample was collected through the microscope using a 100x objective (Nikon MRD01905). The laser was filtered using a $425 \mathrm{~nm}$ longpass filter and the emission was then focused onto an avalanche photodiode (APD, PD050-CTD, Micro Photon Devices). Photon streams were timed by a HydraHarp 400 (Picoquant) in Time-Tagged Time-Resolved (TTTR) mode.

\section{Corresponding Author}

*E-mail: jcaram@chem.ucla.edu.

\section{Author Contributions}

SMT and LAT synthesized and characterized all materials along with help from MLS. The photoluminescence imaging was done by AVS and TLA, and image alignment and processing was done by AJS.

\section{Funding Sources}

This work was supported by the National Science Foundation Career Award No. 1945572. JRC would like to acknowledge the Faculty Career Development Award from the UCLA office of Equity, Diversity, and Inclusion. LAT would like to acknowledge the UCLA Undergraduate Research Center and Honors Program. 


\section{Acknowledgements}

We thank E. Sletten and the Materials Characterization Laboratory at UCLA for instrumentation. We acknowledge the use of instruments at the Electron Imaging Center for NanoMachines supported by NIH (1S10RR23057 to ZHZ) and CNSI at UCLA.

\section{Supporting Information.}

The following files are available free of charge.

Additional experimental details, additional characterization including absorption and photoluminescence,

TEM, reflectance, photoluminescence lifetimes (PDF)

\section{References}

(1) Ithurria, S.; Tessier, M. D.; Mahler, B.; Lobo, R. P. S. M.; Dubertret, B.; Efros, A. L. Colloidal Nanoplatelets with Two-Dimensional Electronic Structure. Nat. Mater. 2011, 10, 936-941, DOI: $10.1038 /$ nmat3145.

(2) Tessier, M. D.; Mahler, B.; Nadal, B.; Heuclin, H.; Pedetti, S.; Dubertret, B. Spectroscopy of Colloidal Semiconductor Core/Shell Nanoplatelets with High Quantum Yield. Nano Lett. 2013, 13, 3321-3328, DOI:10.1021/nl401538n.

(3) Tessier, M. D.; Javaux, C.; Maksimovic, I.; Loriette, V.; Dubertret, B. Spectroscopy of Single CdSe Nanoplatelets. ACS Nano 2012, 6, 6751-6758, DOI:10.1021/nn3014855.

(4) Izquierdo, E.; Robin, A.; Keuleyan, S.; Lequeux, N.; Lhuillier, E.; Ithurria, S. Strongly Confined HgTe 2D Nanoplatelets as Narrow Near-Infrared Emitters. J. Am. Chem. Soc. 2016, 138, 1049610501, DOI:10.1021/jacs.6b04429.

(5) Izquierdo, E.; Dufour, M.; Chu, A.; Livache, C.; Martinez, B.; Amelot, D.; Patriarche, G.; Lequeux, N.; Lhuillier, E.; Ithurria, S. Coupled HgSe Colloidal Quantum Wells through a Tunable Barrier: A Strategy to Uncouple Optical and Transport Band Gap. Chem. Mater. 2018, 30, 40654072, DOI:10.1021/acs.chemmater.8b01028.

(6) Tenney, S. M.; Vilchez, V.; Sonnleitner, M. L.; Huang, C.; Friedman, H. C.; Shin, A. J.; Atallah, T. L.; Deshmukh, A. P.; Ithurria, S.; Caram, J. R. Mercury Chalcogenide Nanoplatelet-Quantum Dot Heterostructures as a New Class of Continuously Tunable Bright Shortwave Infrared Emitters. J. Phys. Chem. Lett. 2020, 11, 3473-3480, DOI:10.1021/acs.jpclett.0c00958.

(7) Tessier, M. D.; Spinicelli, P.; Dupont, D.; Patriarche, G.; Ithurria, S.; Dubertret, B. Efficient Exciton Concentrators Built from Colloidal Core/Crown CdSe/CdS Semiconductor Nanoplatelets. Nano Lett. 2014, 14, 207-213, DOI:10.1021/nl403746p.

(8) Ithurria, S.; Talapin, D. V. Colloidal Atomic Layer Deposition (c-ALD) Using Self-Limiting Reactions at Nanocrystal Surface Coupled to Phase Transfer between Polar and Nonpolar Media. J. Am. Chem. Soc. 2012, 134, 18585-18590, DOI:10.1021/ja308088d.

(9) Shin, A. J.; Hossain, A. A.; Tenney, S. M.; Tan, X.; Tan, L. A.; Foley, J. J.; Atallah, T. L.; Caram, J. R. Dielectric Screening Modulates Semiconductor Nanoplatelet Excitons. J. Phys. Chem. Lett. 
2021, 12, 4958-4964, DOI:10.1021/acs.jpclett.1c00624.

(10) Lin, Y.; Ling, X.; Yu, L.; Huang, S.; Hsu, A. L.; Lee, Y. H.; Kong, J.; Dresselhaus, M. S.;

Palacios, T. Dielectric Screening of Excitons and Trions in Single-Layer MoS2. Nano Lett. 2014, 14, 5569-5576, DOI:10.1021/n1501988y.

(11) Chernikov, A.; Berkelbach, T. C.; Hill, H. M.; Rigosi, A.; Li, Y.; Aslan, O. B.; Reichman, D. R.; Hybertsen, M. S.; Heinz, T. F. Exciton Binding Energy and Nonhydrogenic Rydberg Series in Monolayer WS 2. Phys. Rev. Lett. 2014, 113, 076802, DOI:10.1103/PhysRevLett.113.076802.

(12) Bernevig, B. A.; Hughes, T. L.; Zhang, S. C. Quantum Spin Hall Effect and Topological Phase Transition in HgTe Quantum Wells. Science. 2006, 314, 1757-1761, DOI:10.1126/science.1133734.

(13) He, Y. M.; Clark, G.; Schaibley, J. R.; He, Y.; Chen, M. C.; Wei, Y. J.; Ding, X.; Zhang, Q.; Yao, W.; Xu, X.; Lu, C. Y.; Pan, J. W. Single Quantum Emitters in Monolayer Semiconductors. Nat. Nanotechnol. 2015, 10, 497-502, DOI:10.1038/nnano.2015.75.

(14) Barthelmi, K.; Klein, J.; Hötger, A.; Sigl, L.; Sigger, F.; Mitterreiter, E.; Rey, S.; Gyger, S.; Lorke, M.; Florian, M.; Jahnke, F.; Taniguchi, T.; Watanabe, K.; Zwiller, V.; Jöns, K. D.; Wurstbauer, U.; Kastl, C.; Weber-Bargioni, A.; Finley, J. J.; Müller, K.; Holleitner, A. W. Atomistic Defects as Single-Photon Emitters in Atomically Thin MoS2. Appl. Phys. Lett. 2020, 117, 070501, DOI:10.1063/5.0018557.

(15) Lin, Z.; Liu, Y.; Halim, U.; Ding, M.; Liu, Y.; Wang, Y.; Jia, C.; Chen, P.; Duan, X.; Wang, C.; Song, F.; Li, M.; Wan, C.; Huang, Y.; Duan, X. Solution-Processable 2D Semiconductors for High-Performance Large-Area Electronics. Nat. 2018, 562, 254-258, DOI:10.1038/s41586-0180574-4.

(16) Lin, Z.; Huang, Y.; Duan, X. Van Der Waals Thin-Film Electronics. Nat. Electron. 2019, 2, 378388, DOI:10.1038/s41928-019-0301-7.

(17) Pedetti, S.; Nadal, B.; Lhuillier, E.; Mahler, B.; Bouet, C. C.; Abécassis, B. A.; Xu, X.; Dubertret, B. Optimized Synthesis of CdTe Nanoplatelets and Photoresponse of CdTe Nanoplatelets Films. Chem. Mater 2013, 25, DOI:10.1021/cm4006844.

(18) Yeltik, A.; Delikanli, S.; Olutas, M.; Kelestemur, Y.; Guzelturk, B.; Demir, H. V. Experimental Determination of the Absorption Cross-Section and Molar Extinction Coefficient of Colloidal CdSe Nanoplatelets. J. Phys. Chem. C. 2015, 119, 26768-26775, DOI:10.1021/acs.jpcc.5b09275.

(19) Naeem, A.; Masia, F.; Christodoulou, S.; Moreels, I.; Borri, P.; Langbein, W. Giant Exciton Oscillator Strength and Radiatively Limited Dephasing in Two-Dimensional Platelets. Phys. Rev. B - Condens. Matter Mater. Phys. 2015, 91, 121302, DOI:10.1103/PhysRevB.91.121302.

(20) Olutas, M.; Guzelturk, B.; Kelestemur, Y.; Yeltik, A.; Delikanli, S.; Demir, H. V. Lateral SizeDependent Spontaneous and Stimulated Emission Properties in Colloidal CdSe Nanoplatelets. ACS Nano 2015, 9, 5041-5050, DOI:10.1021/acsnano.5b01927.

(21) Ma, X.; Diroll, B. T.; Cho, W.; Fedin, I.; Schaller, R. D.; Talapin, D. V.; Gray, S. K.; Wiederrecht, G. P.; Gosztola, D. J. Size-Dependent Biexciton Quantum Yields and Carrier Dynamics of QuasiTwo-Dimensional Core/Shell Nanoplatelets. ACS Nano 2017, 11, 9119-9127, DOI:10.1021/acsnano.7b03943.

(22) Li, Q.; Lian, T. Area- and Thickness-Dependent Biexciton Auger Recombination in Colloidal CdSe Nanoplatelets: Breaking the "Universal Volume Scaling Law." Nano Lett. 2017, 17, 31523158, DOI:10.1021/acs.nanolett.7b00587. 
(23) Van Der Stam, W.; Akkerman, Q. A.; Ke, X.; Van Huis, M. A.; Bals, S.; De Mello Donega, C. Solution-Processable Ultrathin Size- and Shape-Controlled Colloidal Cu2- XS Nanosheets. Chem. Mater. 2015, 27, 283-291, DOI:10.1021/cm503929q.

(24) Almeida, G.; Dogan, S.; Bertoni, G.; Giannini, C.; Gaspari, R.; Perissinotto, S.; Krahne, R.; Ghosh, S.; Manna, L. Colloidal Monolayer $\beta$-In2Se3 Nanosheets with High Photoresponsivity. J. Am. Chem. Soc. 2017, 139, 3005-3011, DOI:10.1021/jacs.6b11255.

(25) Dogan, S.; Bielewicz, T.; Cai, Y.; Klinke, C. Field-Effect Transistors Made of Individual Colloidal PbS Nanosheets. Appl. Phys. Lett. 2012, 101, 073102, DOI:10.1063/1.4745786.

(26) Shamsi, J.; Dang, Z.; Bianchini, P.; Canale, C.; Stasio, F. Di; Brescia, R.; Prato, M.; Manna, L. Colloidal Synthesis of Quantum Confined Single Crystal CsPbBr3 Nanosheets with Lateral Size Control up to the Micrometer Range. J. Am. Chem. Soc. 2016, 138, 7240-7243, DOI:10.1021/jacs.6b03166.

(27) Pedetti, S.; Ithurria, S.; Heuclin, H.; Patriarche, G.; Dubertret, B. Type-II CdSe/CdTe Core/Crown Semiconductor Nanoplatelets. J. Am. Chem. Soc. 2014, 136, 16430-16438, DOI:10.1021/ja509307m.

(28) Kelestemur, Y.; Guzelturk, B.; Erdem, O.; Olutas, M.; Erdem, T.; Usanmaz, C. F.; Gungor, K.; Demir, H. V. CdSe/CdSe1-XTex Core/Crown Heteronanoplatelets: Tuning the Excitonic Properties without Changing the Thickness. J. Phys. Chem. C 2017, 121, 4650-4658, DOI:10.1021/acs.jpcc.6b11809.

(29) Dede, D.; Taghipour, N.; Quliyeva, U.; Sak, M.; Kelestemur, Y.; Gungor, K.; Demir, H. V. Highly Stable Multicrown Heterostructures of Type-II Nanoplatelets for Ultralow Threshold Optical Gain. Chem. Mater. 2019, 31, 1818-1826, DOI:10.1021/acs.chemmater.9b00136.

(30) Schlosser, A.; Graf, R. T.; Bigall, N. C. CdS Crown Growth on CdSe Nanoplatelets: Core Shape Matters. Nanoscale Adv. 2020, 2, 4604-4614, DOI:10.1039/d0na00619j.

(31) Khan, A. H.; Bertrand, G. H. V.; Teitelboim, A.; Sekhar M, C.; Polovitsyn, A.; Brescia, R.; Planelles, J.; Climente, J. I.; Oron, D.; Moreels, I. CdSe/CdS/CdTe Core/Barrier/Crown Nanoplatelets: Synthesis, Optoelectronic Properties, and Multiphoton Fluorescence Upconversion. ACS Nano 2020, 14, 4206-4215, DOI:10.1021/acsnano.9b09147.

(32) Antanovich, A. V.; Prudnikau, A. V.; Melnikau, D.; Rakovich, Y. P.; Chuvilin, A.; Woggon, U.; Achtstein, A. W.; Artemyev, M. V. Colloidal Synthesis and Optical Properties of Type-II CdSeCdTe and Inverted CdTe-CdSe Core-Wing Heteronanoplatelets. Nanoscale 2015, 7, 8084-8092, DOI:10.1039/c4nr07134d.

(33) Bouet, C.; Mahler, B.; Nadal, B.; Abecassis, B.; Tessier, M. D.; Ithurria, S.; Xu, X.; Dubertret, B. Two-Dimensional Growth of CdSe Nanocrystals, from Nanoplatelets to Nanosheets. Chem. Mater. 2013, 25, 639-645, DOI:10.1021/cm304080q.

(34) Kurtina, D. A.; Garshev, A. V.; Vasil’Eva, I. S.; Shubin, V. V.; Gaskov, A. M.; Vasiliev, R. B. Atomically Thin Population of Colloidal CdSe Nanoplatelets: Growth of Rolled-up Nanosheets and Strong Circular Dichroism Induced by Ligand Exchange. Chem. Mater. 2019, 31, 9652-9663, DOI:10.1021/acs.chemmater.9b02927.

(35) Ithurria, S.; Bousquet, G.; Dubertret, B. Continuous Transition from 3D to 1D Confinement Observed during the Formation of CdSe Nanoplatelets. J. Am. Chem. Soc. 2011, 133, 3070-3077, DOI:10.1021/ja110046d.

(36) Liu, Y.; Weiss, N. O.; Duan, X.; Cheng, H. C.; Huang, Y.; Duan, X. Van Der Waals 
Heterostructures and Devices. Nat. Rev. Mater. 2016, 1, 1-17, DOI:10.1038/natrevmats.2016.42.

(37) Chhowalla, M.; Jena, D.; Zhang, H. Two-Dimensional Semiconductors for Transistors. Nat. Rev. Mater. 2016, 1, 1-15, DOI:10.1038/natrevmats.2016.52.

(38) Beck, M. E.; Hersam, M. C. Emerging Opportunities for Electrostatic Control in Atomically Thin Devices. ACS Nano 2020, 14, 6498-6518, DOI:10.1021/ACSNANO.0C03299.

(39) Riedinger, A.; Ott, F. D.; Mule, A.; Mazzotti, S.; Knüsel, P. N.; Kress, S. J. P.; Prins, F.; Erwin, S. C.; Norris, D. J. An Intrinsic Growth Instability in Isotropic Materials Leads to Quasi-TwoDimensional Nanoplatelets. Nat. Mater. 2017, 16, 743-748, DOI:10.1038/nmat4889.

(40) Jana, S.; Frutos, M. de; Davidson, P.; Abécassis, B. Ligand-Induced Twisting of Nanoplatelets and Their Self-Assembly into Chiral Ribbons. Sci. Adv. 2017, 3, e1701483, DOI:10.1126/SCIADV.1701483.

(41) Jana, S.; De Frutos, M.; Davidson, P.; Abécassis, B. Ligand-Induced Twisting of Nanoplatelets and Their Self-Assembly into Chiral Ribbons. Sci. Adv. 2017, 3, e1701483, DOI:10.1126/sciadv.1701483.

(42) Cunningham, P. D.; Coropceanu, I.; Mulloy, K.; Cho, W.; Talapin, D. V. Quantized Reaction Pathways for Solution Synthesis of Colloidal ZnSe Nanostructures: A Connection between Clusters, Nanowires, and Two-Dimensional Nanoplatelets. ACS Nano 2020, 14, 12, DOI:10.1021/ACSNANO.9B09051.

(43) Nakonechnyi, I.; Sluydts, M.; Justo, Y.; Jasieniak, J.; Hens, Z. Mechanistic Insights in Seeded Growth Synthesis of Colloidal Core/Shell Quantum Dots. Chem. Mater. 2017, 29, 4719-4727, DOI:10.1021/acs.chemmater.7b00354.

(44) Yang, H.; Hamachi, L. S.; Rreza, I.; Wang, W.; Chan, E. M. Design Rules for One-Step Seeded Growth of Nanocrystals: Threading the Needle between Secondary Nucleation and Ripening. Chem. Mater. 2019, 31, 4173-4183, DOI:10.1021/acs.chemmater.9b00971.

(45) Moreels, I.; Lambert, K.; Smeets, D.; De Muynck, D.; Nollet, T.; Martins, J. C.; Vanhaecke, F.; Vantomme, A.; Delerue, C.; Allan, G.; Hens, Z. Size-Dependent Optical Properties of Colloidal PbS Quantum Dots. ACS Nano 2009, 3, 3023-3030, DOI:10.1021/nn900863a.

(46) Lhuillier, E.; Hease, P.; Ithurria, S.; Dubertret, B. Selective Electrophoretic Deposition of CdSe Nanoplatelets. Chem. Mater. 2014, 26, 4514-4520, DOI:10.1021/cm501713s.

(47) Kodanek, T.; Banbela, H. M.; Naskar, S.; Adel, P.; Bigall, N. C.; Dorfs, D. Phase Transfer of 1and 2-Dimensional Cd-Based Nanocrystals. Nanoscale 2015, 7, 19300-19309, DOI:10.1039/C5NR06221G.

(48) Antanovich, A.; Prudnikau, A.; Matsukovich, A.; Achtstein, A.; Artemyev, M. Self-Assembly of CdSe Nanoplatelets into Stacks of Controlled Size Induced by Ligand Exchange. J. Phys. Chem. C 2016, 120, 5764-5775, DOI:10.1021/acs.jpcc.5b12139.

(49) Ott, F. D.; Riedinger, A.; Ochsenbein, D. R.; Knüsel, P. N.; Erwin, S. C.; Mazzotti, M.; Norris, D. J. Ripening of Semiconductor Nanoplatelets. Nano Lett. 2017, 17, 6870-6877, DOI:10.1021/acs.nanolett.7b03191.

(50) Knüsel, P. N.; Riedinger, A.; Rossinelli, A. A.; Ott, F. D.; Mule, A. S.; Norris, D. J. Experimental Evidence for Two-Dimensional Ostwald Ripening in Semiconductor Nanoplatelets. Chem. Mater. 2020, 32, 3312-3319, DOI:10.1021/ACS.CHEMMATER.0C01238. 
(51) Pun, A. B.; Mazzotti, S.; Mule, A. S.; Norris, D. J. Understanding Discrete Growth in Semiconductor Nanocrystals: Nanoplatelets and Magic-Sized Clusters. Acc. Chem. Res. 2021, 20, 21, DOI:10.1021/acs.accounts.0c00859.

(52) Ji, B.; Giovanelli, E.; Habert, B.; Spinicelli, P.; Nasilowski, M.; Xu, X.; Lequeux, N.; Hugonin, J. P.; Marquier, F.; Greffet, J. J.; Dubertret, B. Non-Blinking Quantum Dot with a Plasmonic Nanoshell Resonator. Nat. Nanotechnol. 2015, 10, 170-175, DOI:10.1038/nnano.2014.298.

(53) Andoy, N. M.; Zhou, X.; Choudhary, E.; Shen, H.; Liu, G.; Chen, P. Single-Molecule Catalysis Mapping Quantifies Site-Specific Activity and Uncovers Radial Activity Gradient on Single 2D Nanocrystals. J. Am. Chem. Soc. 2013, 135, 1845-1852, DOI:10.1021/ja309948y.

(54) Zou, N.; Chen, G.; Mao, X.; Shen, H.; Choudhary, E.; Zhou, X.; Chen, P. Imaging Catalytic Hotspots on Single Plasmonic Nanostructures via Correlated Super-Resolution and Electron Microscopy. ACS Nano 2018, 12, 5570-5579, DOI:10.1021/acsnano.8b01338.

(55) Hayee, F.; Yu, L.; Zhang, J. L.; Ciccarino, C. J.; Nguyen, M.; Marshall, A. F.; Aharonovich, I.; Vučković, J.; Narang, P.; Heinz, T. F.; Dionne, J. A. Revealing Multiple Classes of Stable Quantum Emitters in Hexagonal Boron Nitride with Correlated Optical and Electron Microscopy. Nat. Mater. 2020, 19, 534-539, DOI:10.1038/s41563-020-0616-9.

(56) Dahlberg, P. D.; Perez, D.; Su, Z.; Chiu, W.; Moerner, W. E. Cryogenic Correlative Single-Particle Photoluminescence Spectroscopy and Electron Tomography for Investigation of Nanomaterials. Angew. Chemie 2020, 132, 15772-15778, DOI:10.1002/ANGE.202002856.

(57) Comtet, J.; Glushkov, E.; Navikas, V.; Feng, J.; Babenko, V.; Hofmann, S.; Watanabe, K.; Taniguchi, T.; Radenovic, A. Wide-Field Spectral Super-Resolution Mapping of Optically Active Defects in Hexagonal Boron Nitride. Nano Lett. 2019, 19, 2516-2523, DOI:10.1021/ACS.NANOLETT.9B00178.

(58) Stern, H. L.; Wang, R.; Fan, Y.; Mizuta, R.; Stewart, J. C.; Needham, L.-M.; Roberts, T. D.; Wai, R.; Ginsberg, N. S.; Klenerman, D.; Hofmann, S.; Lee, S. F. Spectrally Resolved Photodynamics of Individual Emitters in Large-Area Monolayers of Hexagonal Boron Nitride. ACS Nano 2019, 13, 4538-4547, DOI:10.1021/ACSNANO.9B00274.

(59) Magnozzi, M.; Pflug, T.; Ferrera, M.; Pace, S.; Ramó, L.; Olbrich, M.; Canepa, P.; Ağircan, H.; Horn, A.; Forti, S.; Cavalleri, O.; Coletti, C.; Bisio, F.; Canepa, M. Local Optical Properties in CVD-Grown Monolayer WS2 Flakes. J. Phys. Chem. C 2021, 125, 16059-16065, DOI:10.1021/ACS.JPCC.1C04287.

(60) Liu, Z.; Amani, M.; Najmaei, S.; Xu, Q.; Zou, X.; Zhou, W.; Yu, T.; Qiu, C.; Birdwell, A. G.; Crowne, F. J.; Vajtai, R.; Yakobson, B. I.; Xia, Z.; Dubey, M.; Ajayan, P. M.; Lou, J. Strain and Structure Heterogeneity in MoS2 Atomic Layers Grown by Chemical Vapour Deposition. Nat. Commun. 2014, 5, 1-9, DOI:10.1038/ncomms6246.

(61) Zhang, K.; Wang, Y.; Joshi, J.; Zhang, F.; Subramanian, S.; Terrones, M.; Vora, P.; Crespi, V.; Robinson, J. A. Probing the Origin of Lateral Heterogeneities in Synthetic Monolayer Molybdenum Disulfide. 2D Mater. 2019, 6, 025008, DOI:10.1088/2053-1583/AAFD9A.

(62) Gutiérrez, H. R.; Perea-López, N.; Elías, A. L.; Berkdemir, A.; Wang, B.; Lv, R.; López-Urías, F.; Crespi, V. H.; Terrones, H.; Terrones, M. Extraordinary Room-Temperature Photoluminescence in Triangular WS2 Monolayers. Nano Lett. 2012, 13, 3447-3454, DOI:10.1021/NL3026357.

(63) Ma, C.; Yan, J.; Huang, Y.; Yang, G. Photoluminescence Manipulation of WS2 Flakes by an Individual Si Nanoparticle. Mater. Horizons 2019, 6, 97-106, DOI:10.1039/C8MH01072B. 
(64) Yan, J.; Ma, C.; Liu, P.; Yang, G. Plasmon-Induced Energy Transfer and Photoluminescence Manipulation in MoS2 with a Different Number of Layers. ACS Photonics 2017, 4, 1092-1100, DOI:10.1021/ACSPHOTONICS.6B00891.

(65) Kim, W. D.; Yoon, D.-E.; Kim, D.; Koh, S.; Bae, W. K.; Chae, W.-S.; Lee, D. C. Stacking of Colloidal CdSe Nanoplatelets into Twisted Ribbon Superstructures: Origin of Twisting and Its Implication in Optical Properties. J. Phys. Chem. C 2019, 123, 9445-9453, DOI:10.1021/ACS.JPCC.8B09987.

(66) Guzelturk, B.; Erdem, O.; Olutas, M.; Kelestemur, Y.; Demir, H. V. Stacking in Colloidal Nanoplatelets: Tuning Excitonic Properties. ACS Nano 2014, 8, 12524-12533, DOI:10.1021/NN5053734. 